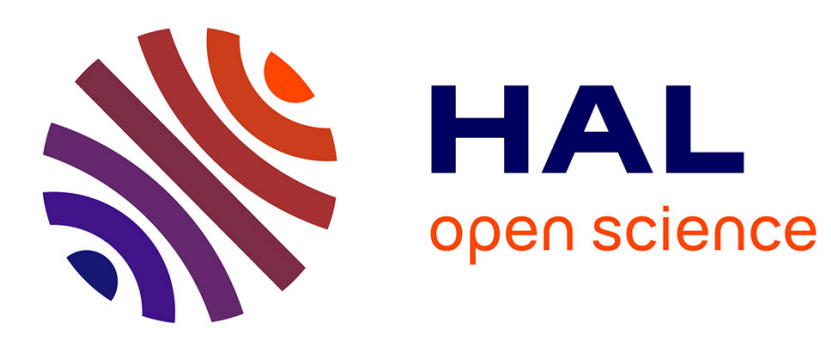

\title{
Forwarder smart selection protocol for limitation of broadcast storm problem
}

\author{
Anna Maria Vegni, Enrico Natalizio
}

\section{To cite this version:}

Anna Maria Vegni, Enrico Natalizio. Forwarder smart selection protocol for limitation of broadcast storm problem. Journal of Network and Computer Applications (JNCA), 2015, 47, pp.61-71. 10.1016/j.jnca.2014.08.009 . hal-01303918

\section{HAL Id: hal-01303918 https://hal.science/hal-01303918}

Submitted on 18 Apr 2016

HAL is a multi-disciplinary open access archive for the deposit and dissemination of scientific research documents, whether they are published or not. The documents may come from teaching and research institutions in France or abroad, or from public or private research centers.
L'archive ouverte pluridisciplinaire HAL, est destinée au dépôt et à la diffusion de documents scientifiques de niveau recherche, publiés ou non, émanant des établissements d'enseignement et de recherche français ou étrangers, des laboratoires publics ou privés. 


\title{
Forwarder Smart Selection Protocol for Limitation of Broadcast Storm Problem
}

\author{
Anna Maria Vegnia,*, Enrico Natalizio ${ }^{\mathrm{b}, \mathrm{b}}$ \\ ${ }^{a}$ Department of Engineering, University of Roma Tre, \\ Via Vito Volterra 62, 00146 Rome, ITALY \\ ${ }^{b}$ Heudiasyc UMR CNRS 7253, Université de Technologie de Compiègne, Compiègne, FRANCE
}

\begin{abstract}
In this paper we introduce a novel routing protocol for efficiently broadcasting packets in vehicular ad hoc networks, while limiting multiple copies of the same packet and avoiding collisions. In high congested traffic scenarios, the increase of packet collisions and medium contentions among vehicles, affects the intervehicular communications and causes a degradation of Quality-of-Service (QoS). The effect also results in a very high number of message copies and collisions within the vehicular network. This is the well-known broadcast storm problem.
\end{abstract}

Our proposed technique, namely Selective Reliable Broadcast protocol (SRB), is intended to limit the number of packet transmissions. Through an opportunistic vehicle selection, packets are retransmitted towards a next hop, in order to strongly reduce the number of forwarder vehicles, while preserving an acceptable level of QoS. SRB belongs to the class of broadcast protocols, as well as cluster-based approaches. It exploits the partitioning behavior, as typical from vehicular ad hoc networks, in order to automatically detect vehicular clusters, intended as zone of

\footnotetext{
*Corresponding author

Email addresses: annamaria.vegni@uniroma 3 . it (Anna Maria Vegni), enrico.natalizio@hds.utc.fr(Enrico Natalizio)
} 
interest. Packets will be then forwarded only to selected vehicles, opportunistically elected as cluster-heads. SRB performance have been assessed in different vehicular scenarios, mostly realistic environments, as urban and highway scenarios. The effectiveness of SRB has been also compared to traditional broadcast protocol, since in respect of traditional broadcasting, the main strengths of SRB are the efficiency of detecting clusters and selecting forwarders in a fast way. The limitation of the broadcast storm problem, as provided by SRB, is expressed in terms of a reduction of number of next-hop forwarders.

Keywords: Vehicular ad hoc networks, opportunistic routing, clusters, broadcast storm problem

\section{Introduction}

Vehicular Ad hoc NETworks (VANETs) are emerging as the preferred network design for Intelligent Transportation Systems (ITS), providing inter-vehicular shortrange communications, for the support of Internet access and safety applications.

VANETs are a particular class of Mobile Ad-hoc NETworks (MANETs), showing typical characteristics. Indeed, VANETs consist of mostly highly mobile nodes moving along the same or opposite directions (i.e., vehicles), forming clusters according to a Poisson distribution, [1]. Communications are supported due to "smart" vehicles, equipped with On-Board Unit with multi-Network Interface Cards (NIC), such as IEEE 802.11p, WiMax, Long Term Evolution, and also GNSS receiver. However, communications among vehicles belonging to different clusters are not always guaranteed, due to connectivity disruptions caused by quick topology network changes, vehicle speed and sparse or totally disconnected scenarios. Moreover, also the market penetration rate can hinder inter-vehicular 
communications: unequipped vehicles physically occupy space and alter the spatial distribution of equipped vehicles and their mobility. On the other side, connectivity improves as the market penetration increases, since it directly translates in an increasing probability of finding a neighboring vehicle that forwards messages.

Inter-vehicle communications are expected to significantly improve transportation safety and mobility on the road. Several applications of inter-vehicle communications have been identified, from safety and warning applications, up to traffic control and driver assistance applications [1]. Many of these applications require multicast routing protocol to a group of vehicles satisfying a geographical criterion.

In this vision, most applications targeting VANETs rely heavily on broadcast transmissions, such as to discover neighboring vehicles, as well as to disseminate traffic-related information to all reachable vehicles within a certain geographical area i.e., mostly in general for context-aware applications. On the other side, broadcasting packets may lead to frequent contention and collisions, due to redundant transmissions among vehicles in dense network topologies (e.g., in rush traffic scenarios). This problem is referred to as the broadcast storm problem [2, 3]. It affects inter-vehicle communications, since redundant rebroadcasts, contention and collisions can be largely increased; when a vehicle rebroadcasts a message, it is highly likely that the neighboring vehicles have already received it, and this results in a large number of redundant messages and replica.

In traditional MANET environment, multiple solutions have been proposed in order to alleviate the broadcast storm effect, but only a few solutions have been addressed to the VANET context $[4,5]$. Most of recent research works have focused on analyzing VANETs as well-connected networks, providing high vehicular traf- 
fic density. As vehicles in close proximity detect the same dangerous situation (e.g., they are approaching a zone-of-relevance), they will inevitably broadcast messages relating to the same event, leading to a dramatically excessive message redundancy. In such scenarios, broadcast suppression solutions have to be considered $[3,6]$. In contrast, in low vehicular traffic density environment, with a sparse RSU settling and a low market penetration rate, vehicular connectivity results intermittent, poor, and short-lived [3]. In this context, the design of reliable and efficient routing protocols for supporting highly diverse and mainly intermittently connected vehicular network topologies is still a challenge.

In this paper, we present a cluster-based broadcast technique for safety applications in VANETs (e.g., car-platoons detection). Our approach is called Selective Reliable Broadcast (SRB), and relies on the opportunistic cluster selection in order to reduce the broadcast storm effect: SRB selects only one vehicle within a cluster-namely, a cluster-head-in order to efficiently rebroadcast emergency and control messages. SRB technique is then able to detect the well-known car platoons, which cause traffic congestions and delays, in a fast way and with low overhead, in order to eventually recommend alternative paths to other vehicles. Comparison with traditional broadcast approach has shown how SRB limits the number of transmissions but preserves good network performance.

This paper is organized as follows. In Section 2 we address pre-existing different solutions for the broadcast storm problem in VANETs. In Section 3, we describe the main phases of SRB technique, and in particular the cluster detection mechanism. In order to enforce the benefit of SRB, we extend the previous work in [7]: in Section 4 we stress the simulation results by a comparison with traditional broadcast protocol. Simulations have been carried out in different scenar- 
ios, showing how SRB provides an automatic cluster detection mechanism, while keeping low the number of forwarder vehicles. Finally, conclusions are drawn in Section 5.

\section{Related Work}

In this section we give an overview of previous contributions in broadcast protocols for VANETs, particularly focusing on cluster-based approaches. Within the discussion, we clarify the paper objective and then introduce our proposed approach.

Reliable protocols use three methods i.e., (i) rebroadcasting, where the transmitter node retransmits the same message for many times, (ii) selective ACK, where the transmitter requires ACK from a small set of the neighbors, and (iii) changing parameters, where the transmitter changes transmission parameters according to the expected state of the network.

The problem statement for reliable protocols is to design a protocol that can deliver a message from a single source to every node in the own transmission range with the highest possible reliability and minimum delay. A successful message dissemination in VANETs needs an efficient decision mechanism in order

to maximize reliability and keep the overhead low. The decision criterion about when and how a safety message should be delivered or repeated is an open issue.

Given the requirements of safety applications (i.e., low delay and effective reliability), and the limitations of vehicular communications (i.e, short-lived connectivity links), selective broadcast or multicast strategies seem more applicable than either unicast routing or flooding. In fact the latter generates a high overhead without increasing the success rate substantially, [8]. Several solutions have been 


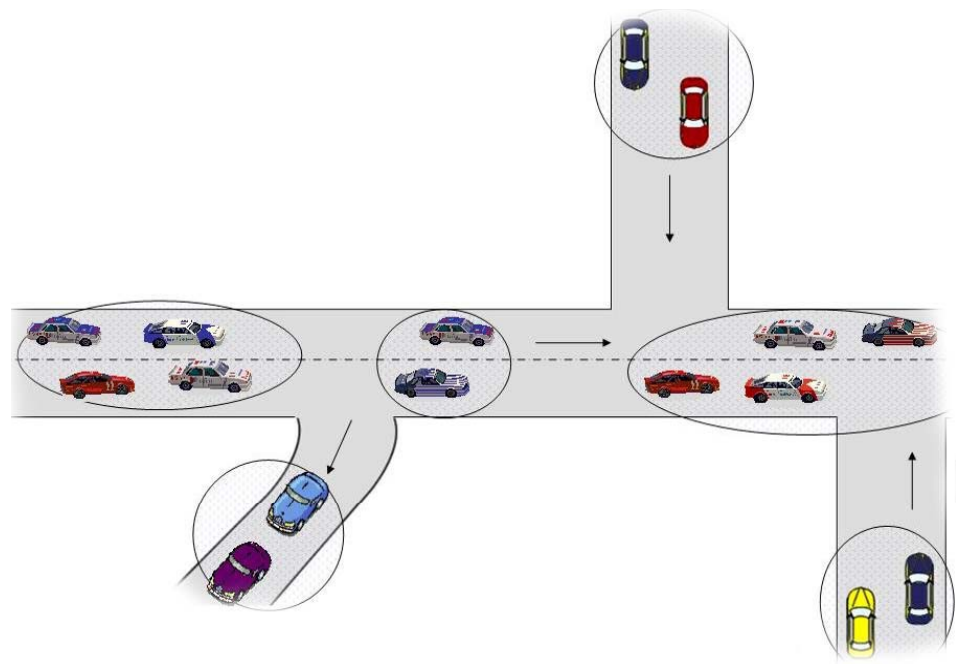

Figure 1: Schematic of several vehicle clusters. Vehicles belonging to the same cluster can communicate each other, while due to gaps among consecutive clusters, no inter-cluster communications are available.

made to introduce intelligence to the basic broadcast concept and make it more selective and, thus, more efficient in its resource usage.

A largely common assumption in connectivity models for VANETs is that a vehicular network is partitioned into a number of clusters [9]; vehicles within a partition can communicate either directly or through multiple hops among each other, but no direct connection exists between partitions, as well depicted in Figure 1. A particular class of routing protocols uses this assumption by exploiting clusters formation, namely cluster-based approaches [10].

Based on geographical locations, directions of movement, speed and many other metrics, vehicles can group into different clusters. Clustering enhances effective broadcasting and relaying of messages, while reducing the overhead associated with signaling and the number of unnecessary message replica. This is due since links among vehicles within the same cluster tend to be more stable, 
although dynamic topology changes can occur. Leveraging on this issue, an efficient clustering should be based on adequate metrics and should take into account the frequent topology changes. The formation of clusters and the selection of the cluster-head (i.e., a vehicle leader within the cluster, responsible for intra and inter-cluster communications) is strongly affected by the high mobility dynamic cluster formation process.

In [11], Ni et al. consider each cluster comprised of three node types: head, gateway and member. The gateway nodes are those who connect to the gateway nodes in other clusters, while the cluster-head is a node whose transmission radius can reach everyone in the same cluster. Finally, members are those who do not belong in either head or gateway group. When a gateway node receives a message from other clusters, it will rebroadcast the message that will be received, and then further retransmitted, by the cluster-head. Although this cluster architecture is correct, the authors did not specify the procedure for the cluster-head election.

In [12], Fasolo et al. propose a Smart Broadcast protocol, which exploits vehicles' positions. The proposed technique assumes that the vehicular network is partitioned in adjacent sectors and that vehicles are able to estimate their own position and, therefore, the sector they belong to. The Smart Broadcast technique considers a contention resolution procedure to elect the relay nodes. Although this technique seems very efficient, it has not been validated in terms of network performance and system overhead. Another work which considers both information on vehicles' position and the cluster formation is presented by Luo et al. [13]. Their approach is a cluster-based routing protocol and the basic idea is to divide the geographic area into foursquare grids, where a vehicle is elected as the clusterhead to route data packets across nearby grids. Also this technique needs to be 
validated via simulation results.

In all previous works, mobility aspects have not been considered, while it is noticeable that the cluster selection process is particularly affected by vehicle mobility and cluster stability. Benslimane et al. [14] consider the cluster formation on the basis of the direction of vehicles movement, the Received Signal Strength (RSS), and the inter-vehicular distance. In this envision, the directional antennabased MAC protocols are exploited to accurately group vehicles on the basis of the direction of their movements and the transmission angles. In [15], Gunter et al. take into account mobility during cluster collision, and a cluster-head vehicle is that one with the lowest relative mobility and closest proximity to its neighbors. Alternatively, in [16] Kayis and Acarman classify nodes into speed groups, so that nodes belonging to the same speed group will be in the same cluster. Finally, a well-known mobility-based clustering technique is MOBIC [17], which considers an aggregate local mobility metric as the basis for cluster formation: the node with the smallest variance of relative mobility to its neighbors is elected as the cluster-head.

In this work, we present $\mathrm{SRB}$, a reliable cluster-based routing protocol that is expected to minimize the number of rebroadcast messages. SRB considers the cluster selection process, and the cluster-head election, by exploiting the intervehicle distance and the time delay. Via simulation results, SRB results in an efficient method to detect clusters and alleviate the broadcast storm problem.

\section{Selective Reliable Broadcast}

The aim of SRB is twofold: (i) to avoid rebroadcast messages (i.e., a limitation of broadcast storm problem), and (ii) to detect clusters in automatic and fast 
way. The proposed approach considers the message rebroadcast process within a VANET by selecting a limited number of vehicles, acting as forwarders. Far from traditional broadcast routing, SRB detects clusters of vehicles in a fast and efficient way, and elects one Cluster-Head $(\mathrm{CH})$ vehicle for each cluster detected. The $\mathrm{CH}$ is then selected as the next message forwarder.

Let us assume a hybrid traffic scenario, consisting of both high and low speed vehicles, as well as high and low vehicle density areas. This scenario well depicts a real vehicular environment, i.e. a clear highway with congested entries. To avoid sudden and emergency braking, information about congested areas should reach high speed approaching vehicles in a timely fashion. We consider an aggregated vehicles group as a Zone-of-Relevance (ZOR) ${ }^{1}$, depicted by the following features i.e., (i) high vehicular density, and (ii) low vehicle speed. We will show how $\mathrm{SRB}$ is able to efficiently discover the ZOR (i.e., with low overhead and delay).

SRB leverages on two main assumptions, i.e. (i) the vehicular area is partitioned in adjacent sectors, and (ii) all vehicles equipped with GPS are able to estimate their own position. Network partitioning (or fragmentation, [9]) is introduced in order to accordingly propagate information messages inside the VANET, while avoiding multiple copies of the same message to the same recipients.

Sectors provide loop-free message propagation along the transmission direction from the source vehicle, and are identified as portions of a circle. The sector size varies as a dynamic process, iteratively hop-by-hop, depending on the transmission direction from each transmitting vehicle (i.e., forward and backward in a highway scenario). The $i$-th sector is identified by an angle $\alpha_{i}(h)$, where $h$ is

\footnotetext{
${ }^{1}$ In this paper we use the term ZOR with the meaning of a congested traffic area.
} 
the index of current hop. Initially, in the $h=1$ hop, there is a unique sector that includes the source vehicle's transmission range i.e., corresponding to an angle $\alpha(h)=360^{\circ}$. On the next hops (i.e., when $h>1$ ), $n$ sectors are identified, each one associated to the $n$-th forwarder vehicle, so that the following equation holds

$$
\sum_{i=1}^{n} \alpha_{i}(h=1)=360^{\circ}
$$

where $\alpha_{i}(h=1)$ is the $i$-th angle of the Direction-of-Arrival of each message transmitted by the source vehicle during the first hop. Notice that in order to avoid unnecessary message transmissions -message replications-, by the second hop $(h=2)$ we consider that each forwarder vehicle has an angle corresponding to its forward or backward transmission range, so that $\alpha(h=2)=180^{\circ}$. Figure 2 depicts the sector identification in the first and second hop.

SRB considers a contention resolution procedure necessary to detect the ZOR and nearby clusters, and then elects relay nodes, each per single cluster. The contention procedure and cluster detection mechanism are detailed as follows:

- RTB transmission: A source vehicle transmits an Request-to-Broadcast (RTB) control message to all neighboring vehicles in the transmission range. The RTB is a MAC-broadcast packet that contains the geographical position of the transmitter node. The RTB message size is 7.5 byte, (see Table 1 ). In the first hop, the message propagation direction is omnidirectional, while it changes for the next hops;

- Waiting time calculation: Upon receiving an RTB, vehicles compute their distance from the source vehicle (i.e., $d[\mathrm{~m}])$. The distance information is exploited in the calculation of the waiting time (i.e., $t_{w}[\mathrm{~s}]$ ) 一 a backoff 
time-which depends on maximum and minimum contention window, as follows:

$$
t_{w}=\left[\frac{r_{t x}-d}{r_{t x}} \cdot\left(C W_{\max }-C W_{\min }\right)+C W_{\min }\right] \cdot t_{\text {slot }},
$$

where $r_{t x}[\mathrm{~m}]$ is the transmission range, and $C W_{\max , \min }$ are the maximum and minimum contention window sizes, respectively. By using (2) vehicles in the further regions always transmit before the others. According to the CSMA/CA policy of IEEE 802.11, the backoff time is decremented by 1 at each idle slot, while the decrease is stopped when the medium is busy;

- CTB transmission: Whenever the waiting time associated to a vehicle countdowns to zero, the vehicle sends back to the source a Clear-to-Broadcast (CTB) packet, containing the vehicle ID and its distance from the source. We set the CTB message size to 7.5 byte, (see Table 1) After receiving a valid CTB packet, vehicles exit the contention phase; in case of collisions, vehicles remain in the contention phase and resume the backoff process;

- Cluster detection: The source vehicle receives information on the ID and the distance from its nearby vehicles. By measuring the DoA of the CTB messages, the source vehicle is able to calculate all the mutual inter-vehicle distances among its nearby vehicles. If the distance between each couple of nearby vehicles is lower than a threshold value (i.e., $\left.D_{\min }\right)$, the two vehicles will be considered belonging to the same cluster. The choice of $D_{\min }$ influences the number of clusters identified: the higher the distance threshold, the higher the number of vehicles in each cluster. When $0 \leftarrow D_{\min }$, each vehicle identifies a 1-size cluster; 
Table 1: Message exchanged classification and content.

\begin{tabular}{c|c|c}
\hline \hline Message & Content & Size [byte] \\
\hline RTB & Source node's position & 7.5 \\
\hline CTB & Source-Destination distance & 7.5 \\
\hline Data & CH ID, data information & 1526 \\
\hline \hline
\end{tabular}

- Cluster-head election: After detecting multiple clusters, the source vehicle elects the furthest vehicle inside each cluster as the Cluster-Head, and transmits a data message only to such vehicle. We assume that the data message has a length of 1526 byte, (see Table 1). Upon receiving the data message, each $\mathrm{CH}$ will become the message source for the next contention phase, and the SRB algorithm is repeated for the next hops.

In Table 1 we report the main messages exchanged in the SRB technique. Figure 3 depicts the main phases of SRB technique, for a forward data transmission along the vehicular grid. Once the $\mathrm{CH}$ vehicles are identified, they will forward messages according to SRB protocol.

The design and implementation of the cluster detection mechanism in the SRB algorithm follows the steps of the method ClusterDetection(), as shown in the Algorithm $1^{2}$. Let us define:

- veh, as a $m \times 1$ array whose elements are the vehicles' IDs reachable by the transmitter vehicle. The array is sorted according to the angles formed by the DoA of the messages sent to the transmitter;

\footnotetext{
${ }^{2}$ The Algorithm 1 only represents the main scheme for cluster detection used in the SRB technique, but it is not that one used in our simulations
} 
- sels, as a dynamic array, initially null, whose elements are the forwarder vehicles' IDs (i.e., the selected vehicles for next hop forwarding).

During the initialization, we define three indexes i.e., $i$ and $j$ used to calculate the inter-vehicle distances, and $k$ is the index associated to the array of forwarders (i.e., sels). The method ClusterDetection has a while command, which considers three different cases:

- Case 1: the index $j$ is greater than the number of elements comprised in veh;

- Case 2: the distance between a couple of vehicles is less than $R$ i.e., the minimum distance among clusters;

- Case 3: the distance between a couple of vehicles is greater than $D_{\min }[\mathrm{m}]$. The calculus of distances between two neighboring vehicles $v_{i, j}$ is done by means of the method distance $\left(v_{i}, v_{j}\right)$, and starts for a null value of angle. This means that the first comparison will be between the vehicle with index 0 and the vehicle with index 1 , where 1 and 0 are the positions of elements of the array.

Each time that the distance is less of that one selected, the index $j$ increases, while $i$ is unchanged (i.e., we obtain $j=2$ and $i=0$ ). This represents the Case 1 in the above list. Notice that the value of MaxDist has to be lower than the threshold $D_{\min }$. In the Case 2, when the inter-vehicle distance is higher than that one selected, the following condition holds

$$
j \geq(i+1) .
$$

When $j=(i+1)$, it is easy to understand that a new cluster has been detected, since all the possible combinations of $i$ and $j$ have been proven in order to verify 
the availability of a third vehicle whose distance from the vehicle $j$ is less than the threshold. When $j>(i+1)$, it is still possible to find other combinations of $i$ and $j$ (by increasing $i$ ), in order to assess if the cluster is a new cluster. An example of using the cluster detection mechanism is described in Figure 4.

Finally, in the next performance evaluation section, we will show that our technique delivers data with reasonable delay to most of the intended vehicles. Moreover, the amount of transmitted packets per hop depends on the number of selected vehicles as forwarders, and it is expressed as:

$$
p k t / h o p=\sum_{i=1}^{N}\left(2 n_{i}+1\right),
$$

where $N$ is the number of clusters detected by a source node per hop, and $n_{i}$ is the number of vehicles comprising the $i$-th cluster. The terms $2 n_{i}$ and 1 represent respectively the maximum number of packets RTB and CTB, and one data packet, transmitted in each hop ${ }^{3}$.

\section{Simulation Results}

In this section, we analyze the performance of the proposed technique in two different cases i.e., (i) urban, and (ii) highway scenario. For urban scenario, we generate a 4 kilometers square grid with one lane per direction. In highway scenario, we select a 4 kilometers square area comprised of straight roads with 3 lanes per direction and several entries.

In both scenarios, we assume there are some ZORs, as previously defined, in unknown areas. Packets are generated with a constant generation rate (i.e.,

\footnotetext{
${ }^{3}$ Eq. (4) represents an ideal scenario where drop packets and any collisions are omitted, or at least minimized.
} 
Table 2: Simulation Parameters

\begin{tabular}{l|l}
\hline \hline Parameter & Value \\
\hline Simulation Duration & {$[100,300] \mathrm{s}$} \\
\hline IEEE $802.11 b$ Transmission Range $\left(r_{t x}\right)$ & $300 \mathrm{~m}$ \\
\hline Data Rate $(R)$ & $6 \mathrm{Mbps}$ \\
\hline$D_{\min }$ & $150 \mathrm{~m}$ \\
\hline \hline
\end{tabular}

$\lambda[\mathrm{pck} / \mathrm{s}])$, and are transmitted according to a fixed data rate within a fixed transmission range. All vehicles are equipped with a GPS receiver and radio interfaces, compliant with the IEEE $802.11 b$ standard. Table 2 collects the main parameters used in the simulations.

In order to assess the effectiveness of the proposed SRB protocol, we compare network performance with traditional broadcast approach, since SRB rises from this protocol.

\subsection{Urban scenario}

The urban scenario has been generated through the VanetMobiSim software [18], considering a 4 kilometers square grid, as shown in Figure $5(a)$. Performance have been evaluated in terms of (i) throughput, (ii) number of elected $\mathrm{CHs,} \mathrm{(iii)}$ message propagation, and (iv) end-to-end delay, by means of numerical simulations carried out with the ns-2.34 simulator [20]. In this scenario, we assume 150 vehicles are moving at constant speeds, and forming clusters only for a limitedshort-life-time interval.

The SRB performance mainly depends on the value of the distance threshold $D_{\text {min }}$, since this parameter affects the number of vehicles in a cluster. As a 
consequence, the higher the distance threshold, the lower the number of clusters detected, or equally, the number of $\mathrm{CH}$ vehicles. Figure 7 depicts the percentage of $\mathrm{CHs}$ for different values of $D_{\min }$. Notice that for a low value of distance threshold the SRB is not able to select a limited number of $\mathrm{CHs}$, and approximates the behavior of a traditional broadcast protocol (i.e., all neighboring vehicles are involved in data forwarding). In our simulations, we have considered $D_{\min }=150 \mathrm{~m}$, which represents a good tradeoff with the transmission range (i.e., $\left.D_{\min }=r_{t x} / 2\right)$.

Figure 6 depicts the average throughput [bit/s] experienced by vehicles communicating each others, in the case of SRB and traditional broadcast. During the simulation time, the vehicle cluster aggregation occurs in a random fashion, due to the not homogeneous nature of the urban scenario (i.e., the presence of junctions and traffic lights can reduce cluster formation). Leveraging on this consideration, we can observe a variable behavior of throughput experienced in SRB technique, as shown in Figure $6(a)$ : in the first part of the simulation (i.e., for $t<50 \mathrm{~s}$ ), the throughput shows high values and reaches $30 \mathrm{kbps}$, while in the second part (i.e., for $t \geq 50 \mathrm{~s}$ ) low values of throughput occur. This is justified by an increase of data exchange which occurs for $t<50 \mathrm{~s}$, when the cluster detection occurs; while for $t \geq 50 \mathrm{~s}$ vehicles aggregation in clusters is reduced and traffic flow becomes uncongested.

By comparing results in Figure $6(a)$ and $(b)$, we notice that SRB protocol reaches peaks of $30 \mathrm{kbps}$, while for vehicles broadcasting packets the performance are limited to $5 \mathrm{kbps}$ that could appear as a quite counterintuitive result. Basically, this is due since for a simple broadcast approach the vehicles forward messages to all neighboring, causing multiple copies of the same message, as well as higher 
collision probability. On the other side, SRB allows only a limited number of vehicles to retransmit packets, opportunistically selected, and then reducing the collision probability, as well as the number of message replica. In the broadcast case, the low value of average throughput is due only to the effective transmitted messages, without multiple copies and the collided ones. This result can be better analyzed together with the number of elected $\mathrm{CH}$ vehicles, giving information about the effectiveness of SRB protocol. Compared to traditional broadcast, the SRB selects only the $45 \%$ of vehicles as $\mathrm{CHs}$ and reaches an average throughput of $13 \mathrm{kbps}$. The resource usage is then limited.

In Figure $8(a)$ and $(b)$ is depicted the average message propagation [m], for SRB and broadcast protocols. Data messages for SRB propagate in the network and reach on average long distances (i.e., up to $1.3 \mathrm{~km}$ far from the source); the vehicular environment is then almost fully "covered". It is also important to notice that performance trend reaches high values since a few seconds from the beginning of the simulation. These results suggest that the vehicular connectivity is largely guaranteed in the network. On the other hand, in Figure $8(b)$ for traditional broadcast the message propagation shows low values (i.e., $<300 \mathrm{~m}$ corresponding to the maximum one-hop size). Again, this result is due mainly to the number of collisions and the packet drop probability; for each hop, packets are transmitted by a source vehicle to all the neighbors, resulting as a broadcast storm.

Finally, we show the comparison between SRB and traditional broadcast in terms of average end-to-end delay [s], versus the simulation time. In the case of SRB, Figure 9 (a) depicts how on average the delay is lower than $400 \mathrm{~ms}$, which is particularly suitable for safety applications. Some delay peaks occur, due to multi-hop message propagation time, and the waiting time parameter, while the 
processing time to select $\mathrm{CH}$ s has been considered negligible. For the broadcast approach, as expected, the end-to-end delay is very short, since the message propagation is reduced to one hop propagation due to collisions and packet losses of broadcast storm effect. Figure $9(b)$ depicts the end-to-end delay, basically corresponding to one-hop delay for broadcast approach.

All previous simulation results have shown that in urban scenarios, where vehicle density is not uniform and can limit the cluster formation, SRB provides high message propagation distance with low end-to-end delay, as well as an effective throughput. SRB overcomes traditional broadcasting techniques, and limits the effect of broadcast storming (i.e., collisions, message replica and packet losses).

\subsection{Highway scenario}

The second scenario, shown in Figure $5(b)$, is based on a real urban map of a square-shaped portion of the city of Rome (Italy), with an area equal to $15 \mathrm{~km}^{2}$. The map has been retrieved from the website of the Open Street Map (OSM) [21]. The scenario represents a portion of GRA (Grande Raccordo Anulare, literally "Great Ring Road"), that is a toll-free, ring-shaped orbital motorway encircling Rome. GRA is one of the most important roads in Rome, and traffic reaches up to 160.000 vehicles per day. GRA features up to 42 junctions and in rush hours traffic congestions affect the road condition.

In such scenario we validated the SRB technique works very efficiently to detect clusters in an automatic and fast way, so that vehicles can avoid traffic congestions, and potentially are rerouted to different paths. The simulated scenario consists of a congested area with at least 15 vehicles driving in a platoon at low speed, while 70 vehicles drive into a nearby free area at high speed. Due to the huge area taken into account, we consider a simulation time of $300 \mathrm{~s}$, in order to 
allow vehicles to move and communicate each others.

A vehicle coming from the free area should be able to fast detect the traffic congestion when approaching. Unlikely the urban scenario where vehicles form several clusters randomly, in highway environment we consider a huge cluster, depicted by low vehicle speed and high vehicle density. In the simulations we used a traffic simulator called Simulation of Urban Mobility (SUMO) [19] to generate traffic scenarios, and the trace files have been simulated on the network simulator ns-2.34.

In this section we show the most significant result, which allow to detect clusters in SRB approach i.e., (i) the average throughput. Again, the comparison to the broadcast protocol highlights the effectiveness of SRB.

Figure $10(a)$ and $(b)$ show the average throughput, respectively for SRB and broadcast protocol, versus the simulation time. In both cases, we notice an increase of throughput for $t \geq 175 \mathrm{~s}$, when both SRB and broadcast protocol reach on average $2.0 \mathrm{kbps}$. This behavior is justified since at $t=175 \mathrm{~s}$ a vehicle moving in the highway approaches the congested area, and then an high number of data messages are exchanged. However, due to the nature of highway scenario, with a huge cluster of vehicles, the average value of throughput is lower than that in urban scenario. The initial peaks experienced with the broadcast protocol can be justified because of the increasing number of messages exchanged within the transmission range, which occurs at the beginning of the simulation.

\section{Conclusions}

In this paper we have addressed to recent efforts for mitigating the broadcast storm problem in VANETs. The Selective Reliable Broadcast (SRB) allows 
vehicles to selectively transmit messages within their own transmission range, resulting in a reduction of network overload, and limitation of message duplication. SRB is particularly effective for safety applications: it relies on cluster-based routing protocols, as well as exploits the vehicles' positions, in order to detect traffic congestions (i.e., car platoons) in a fast way and with low overhead. Only a lim-

ited number of vehicles are elected as cluster-heads to forward messages. This allows to save network resources while keeping high performance.

SRB has been validated through extensive simulations, both in urban and highway scenarios, compared to traditional broadcast protocol. Results have shown that the proposed approach is able to detect vehicle clusters, and provides high distances for message dissemination. Especially in highway scenarios, SRB works efficiently by means of detecting congestion areas in a fast way.

\section{References}

[1] H. Hartenstein, K. Laberteaux, VANET: Vehicular Applications and Internetworking Technologies, Wiley \& sons, Ltd., 2010.

[2] O. Tonguz, N. Wisitpongphan, F. Bai, P. Mudalige, V. Sadekar, Broadcasting in VANET, in: Proc. of Mobile Networking for Vehicular Environments, 2007, pp. 7-12.

[3] N. Wisitpongphan, O. Tonguz, J. Parikh, P. Mudalige, F. Bai, V. Sadekar, Broadcast storm mitigation techniques in vehicular ad hoc networks, IEEE Wireless Communications 14 (6) (2007) 84-94.

[4] K. Bür, M. Kihl, Evaluation of Selective Broadcast Algorithms for Safety 
Applications in Vehicular Ad Hoc Networks, International Journal of Vehicular Technology 2011 (2011) 13.

[5] O. Tonguz, N. Wisitpongphan, J. Parikh, F. Bai, P. Mudalige, V. Sadekar, On the Broadcast Storm Problem in Ad hoc Wireless Networks, in: Proc. of 3rd Intl. Conf. on Broadband Communications, Networks and Systems, BROADNETS, 2006, pp. 1-11.

[6] M. Koubek, S. Rea, D. Pesch, Event Suppression for Safety Message Dissemination in VANETs, in: Proc. of IEEE 71st Vehicular Technology Conference (VTC 2010-Spring), 2010, pp. 1-5.

[7] A.M.Vegni, A. Stramacci, E. Natalizio, SRB: A Selective Reliable Broadcast Protocol for Safety Applications in VANET, in: Proc. of Intl. Conf. on Selected Topics in Mobile \& Wireless Networking (iCOST 2012), Avignon, France, 2012, pp. 89-94.

[8] W. Chen, R. K. Guha, T. J. Kwon, J. Lee, I. Y. Hsu, A survey and challenges in routing and data dissemination in vehicular ad-hoc networks, in: Proc. of IEEE International Conference on Vehicular Electronics and Safety, Columbus, Ohio, USA, 2008, pp. 328-333.

[9] T.D.C.Little, A. Agarwal, An Information Propagation Scheme for Vehicular Networks, in: Proc. of IEEE Intelligent Transportation Systems Conference, Vienna, Austria, 2005, pp. 155-160.

[10] J. Kakarla, S. Sathya, G. B. Laxmiand, R. B. Babu, A Survey on Routing Protocols and its Issues in VANET, International Journal of Computer Applications 28 (4) (2011) 38-44. 
[11] S. Ni, Y. Tseng, Y. Chen, J. Sheu, The broadcast storm problem in a mobile ad hoc network, in: Proc. of the 5 th annual ACM/IEEE Intl. Conf. on Mobile Computing and Networking (MobiCom), 1999, pp. 151-162.

[12] E. Fasolo, A. Zanella, M. Zorzi, An Effective Broadcast Scheme for Alert Message Propagation in Vehicular Ad hoc Networks, in: Proc. of IEEE International Conference on Communications (ICC), Vol. 9, 2006, pp. 39603965.

[13] Y. Luo, W. Zhang, Y. Hu, A New Cluster Based Routing Protocol for VANET, in: Proc. on 2nd Intl. Conf. on Networks Security Wireless Communications and Trusted Computing (NSWCTC), Vol. 1, 2010, pp. 176-180.

[14] A. Benslimane, T. Taleb, R. Sivaraj, Dynamic Clustering-Based Adaptive Mobile Gateway Management in Integrated VANET-3G Heterogeneous Wireless Networks, IEEE Journal on Selected Areas in Communications 29 (3) (2011) 559-570.

[15] Y. Gunter, B. Wiegel, H. Grossmann, Cluster-based Medium Access Scheme for Vanets, in: Proc. on Intelligent Transportation Systems Conference, 2007.

[16] O. Kayis, T. Acarman, Clustering formation for inter-vehicle communication, in: Proc. on IEEE Intelligent Transportation Systems Conference (ITST), 2007, pp. 636-641.

[17] P. Basu, N. Khan, T.D.C.Little, A mobility based metric for clustering in mobile ad hoc networks, in: Proc. on International Conference on Distributed Computing Systems Workshop, 2001, pp. 413-418. 
[18] VanetMobiSim, website: http://vanet.eurecom.fr/.

[19] SUMO project, website: http://sourceforge.net/projects/sumo/.

[20] Network simulator 2 (ns-2), available at: http://isi.edu/nsnam/ns/.

[21] Open street map, website: http://www.openstreetmap.org. 


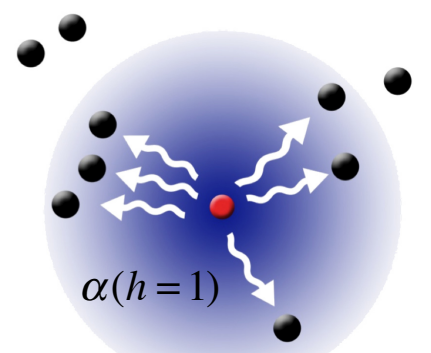

(a)

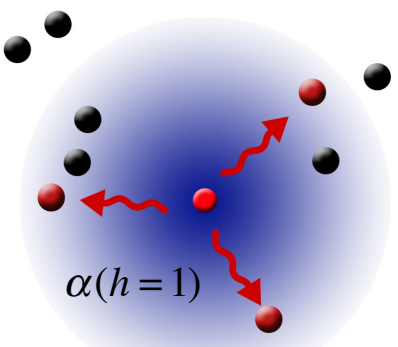

$(c)$

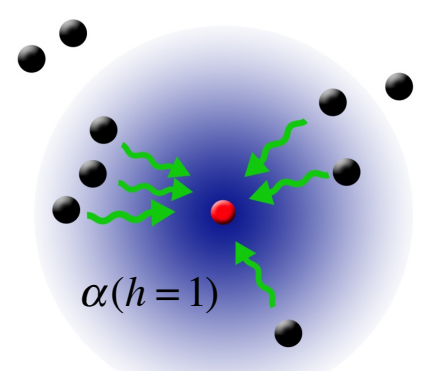

(b)

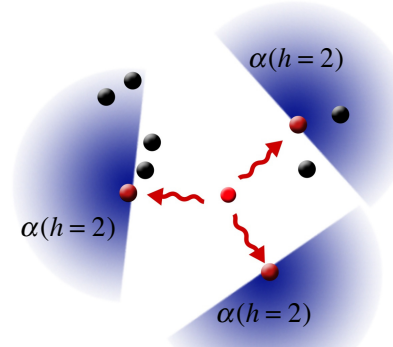

$(d)$

Figure 2: Sector formation process in SRB technique. The red and black points are respectively the source and neighboring vehicles, while the blue area represents the transmission range of one vehicle. In the first hop, during (a) RTB transmission, (b) CTB transmission, and (c) cluster-head selection, $\alpha(h=1)=360^{\circ}$. By the second hop, $(d)$ the message propagation is only backward or forward, and then $\alpha(h=2)=180^{\circ}$. 


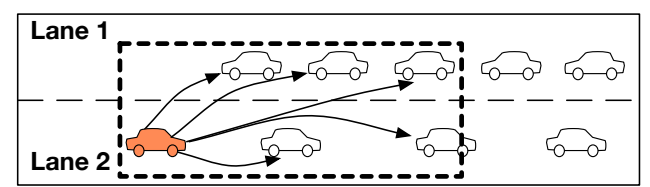

(a)

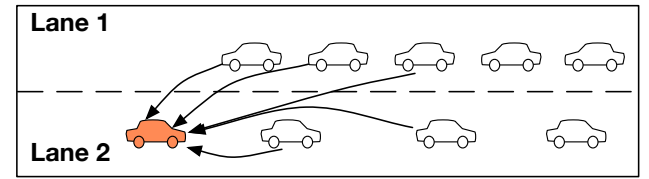

(b)

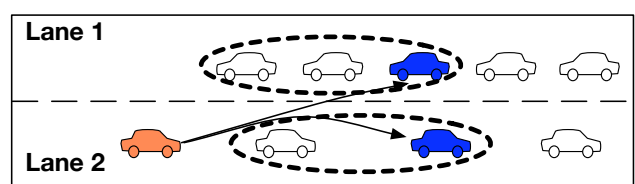

(c)

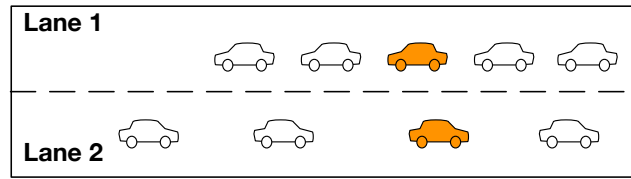

$(d)$

I--I Transmission range to Source/forwarder vehicle

:-- Cluster detected

Figure 3: Main phases of SRB technique: (a) RTB transmission, (b) CTB transmission, (c) Cluster detection and $\mathrm{CH}$ election, and $(d)$ message propagation.

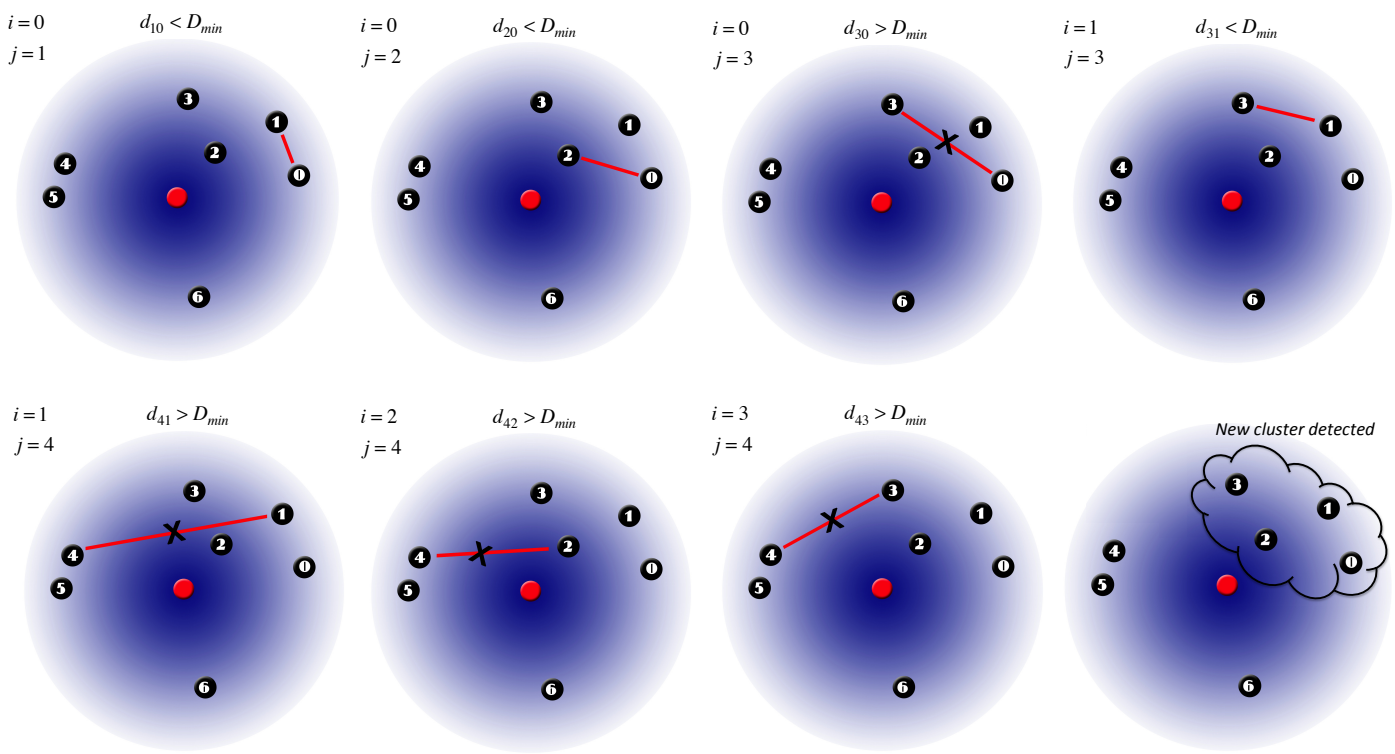

Figure 4: Cluster detection mechanism implemented in SRB technique. From left to right, each vehicle computes the inter-distance among neighbors (i.e., $d_{i j}$ ) and compares it to the threshold (i.e., $\left.D_{\min }\right)$, till the cluster is detected. 


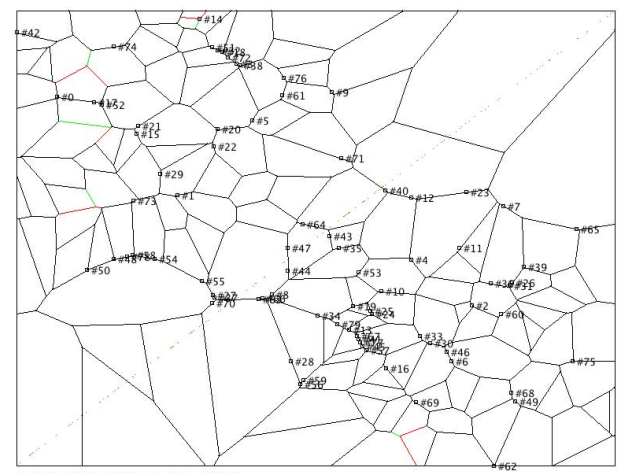

$(a)$

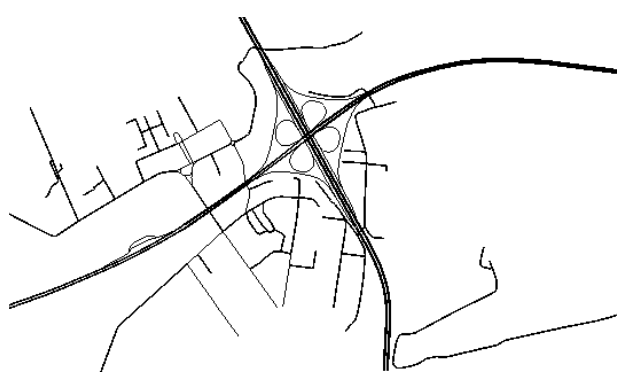

(b)

Figure 5: Simulated scenarios for the assessment of SRB performance. (a) Urban scenario generated with VanetMobiSim [18], and (b) highway scenario (i.e., a selected portion of GRA, Rome Italy) generated with SUMO [19]. 
Method: ClusterDetection()

Initialization:

$i=0$;

$j=1$;

$k=0$;

$\max =i$;

MaxDist $=$ distance $(\operatorname{veh}[i], \mathbf{v e h}[j])$;

go_ahead = true;

while go_ahead do

if $j>m-1$ then

go_ahead $=$ false ;

else

$j++$;

if $\operatorname{distance}(\mathbf{v e h}[i], \mathbf{v e h}[j])>$ MaxDist then

MaxDist $=\operatorname{distance}(\mathbf{v e h}[i], \mathbf{v e h}[j])$;

$\max =\operatorname{veh}[j] ;$

else

if $j==(i+1)$ then

$\operatorname{sels}[k]=\operatorname{veh}[i]$

MaxDist $=\operatorname{distance}(\operatorname{veh}[i], \mathbf{v e h}[j])$;

$\max =j$

$i++; j++; k++$

else

| $i++$;

end

end

$i++$;

end

end

Algorithm 1: Cluster detection algorithm implemented in SRB method. 


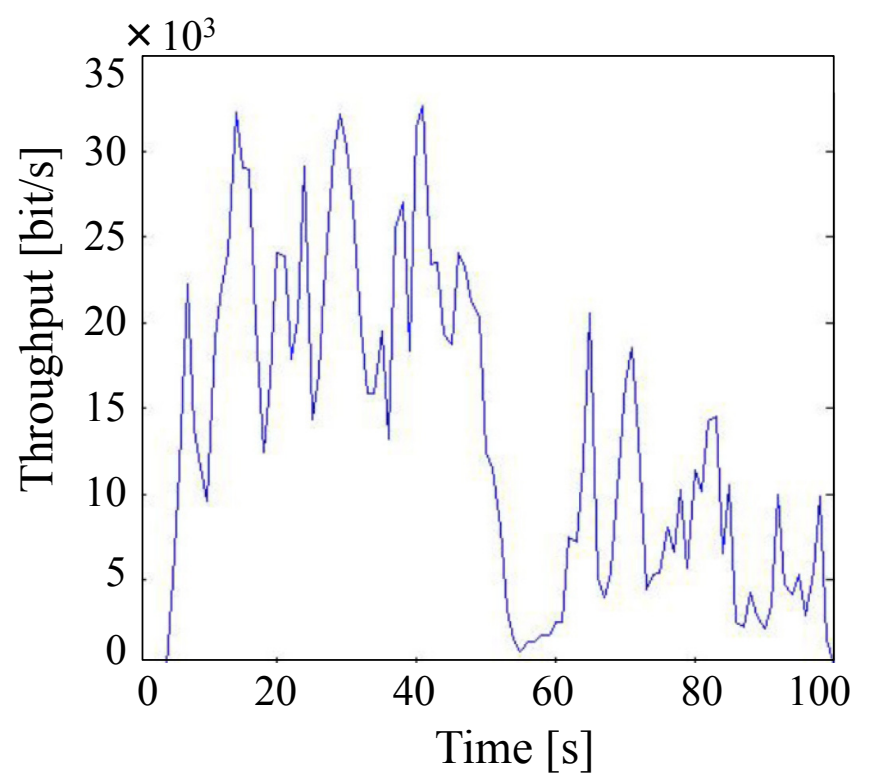

(a)

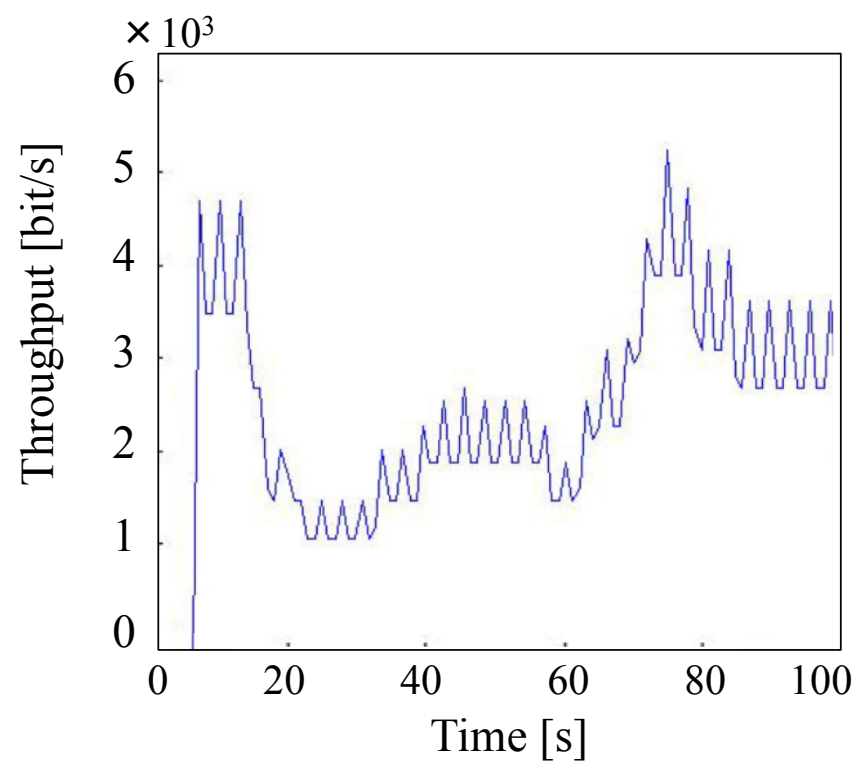

(b)

Figure 6: Urban scenario. Average throughput for (a) SRB technique, and (b) broadcast approach, vs. simulation time. 

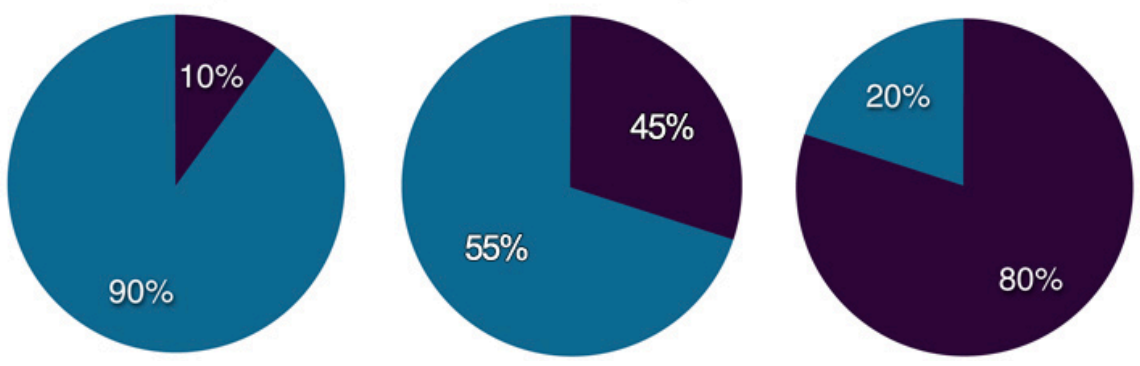

Figure 7: Percentage of selected $\mathrm{CH}$ vehicles (dark blue) for different values of distance threshold. From left to right, $D_{\min }=250 \mathrm{~m}, 150 \mathrm{~m}$, and $50 \mathrm{~m}$. The rest of vehicles (light blue) is not involved into the message dissemination process. 


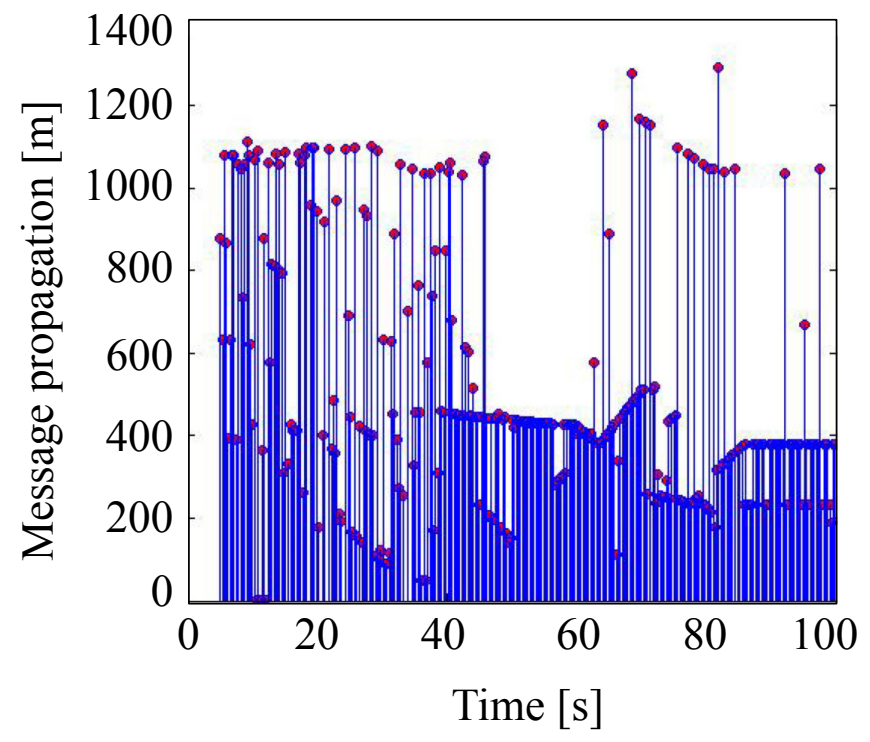

(a)

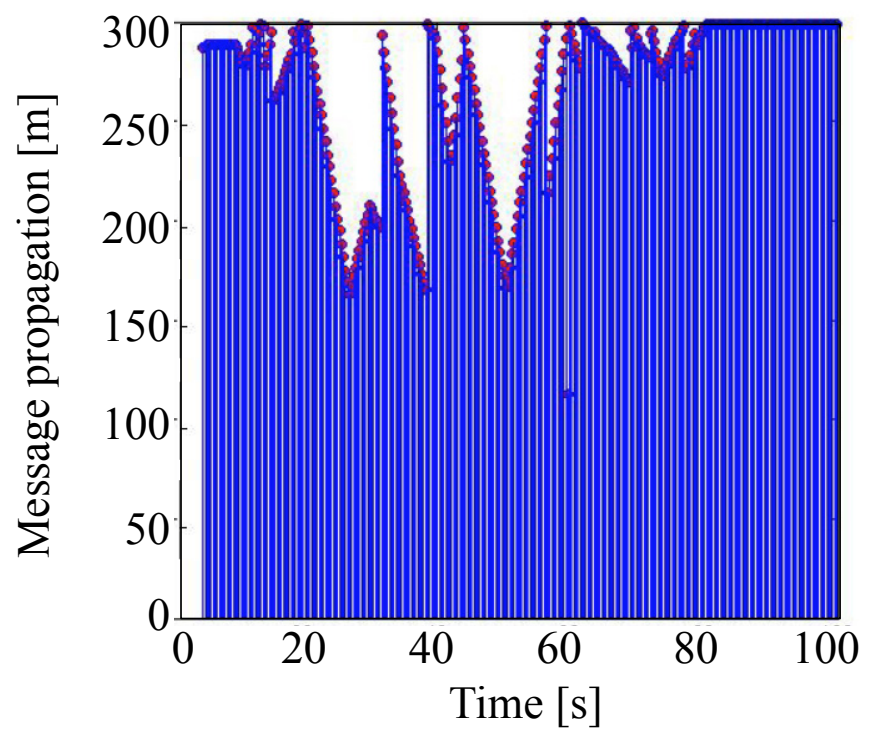

(b)

Figure 8: Urban scenario. Average message propagation for (a) SRB technique, and (b) broadcast approach, vs. simulation time. 


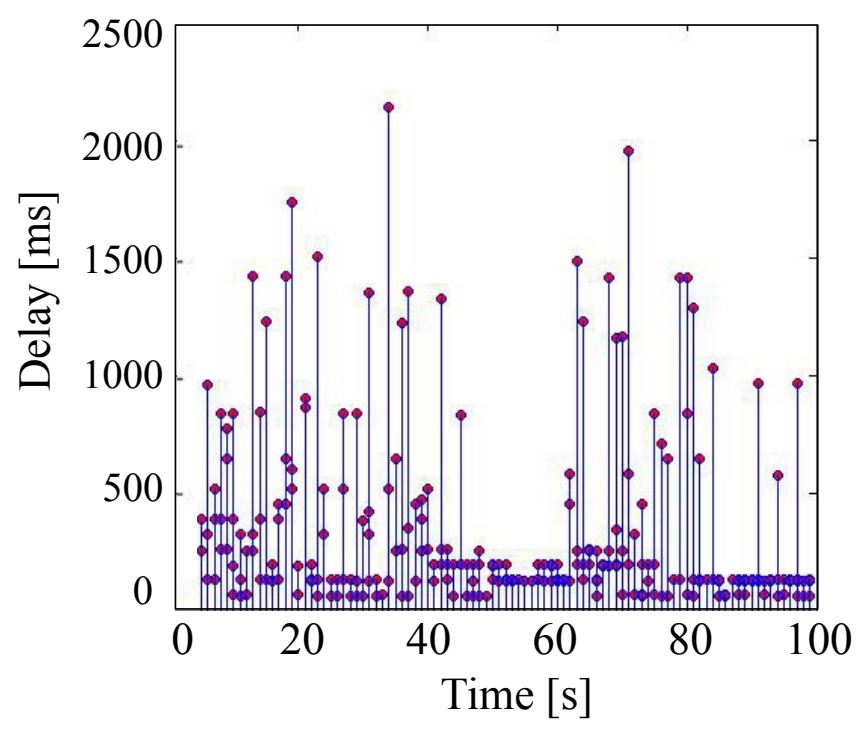

(a)

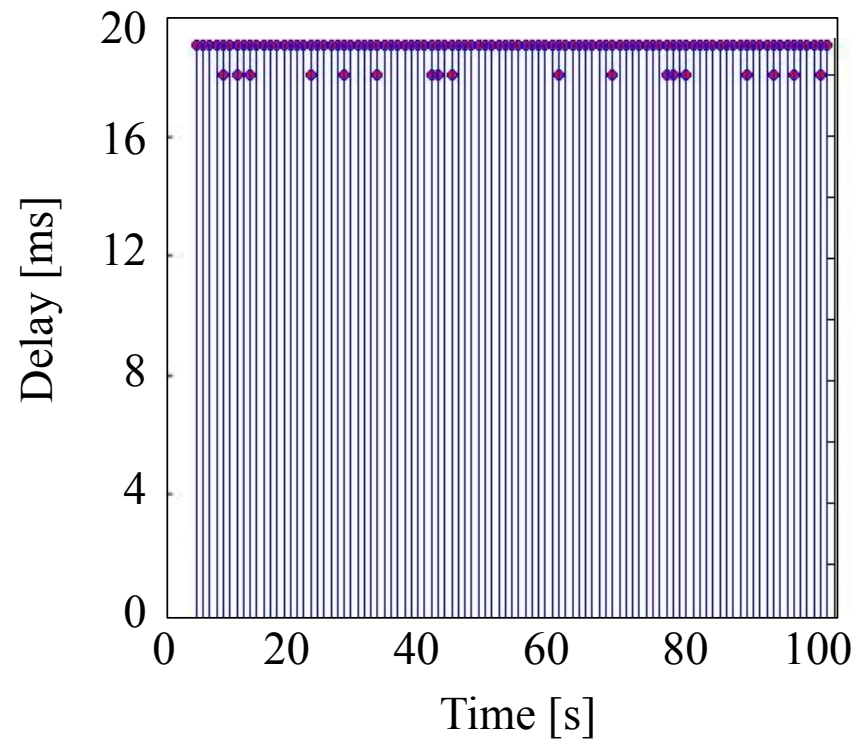

(b)

Figure 9: Urban scenario. Average end-to-end delay for (a) SRB technique, and $(b)$ broadcast approach, vs. simulation time. 


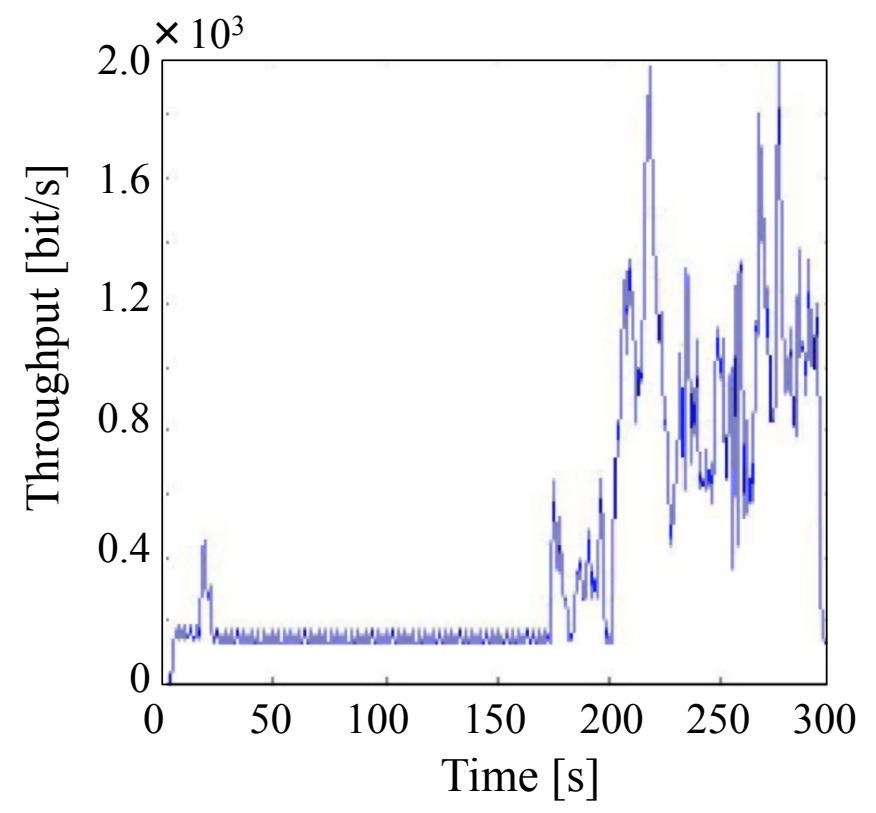

$(a)$

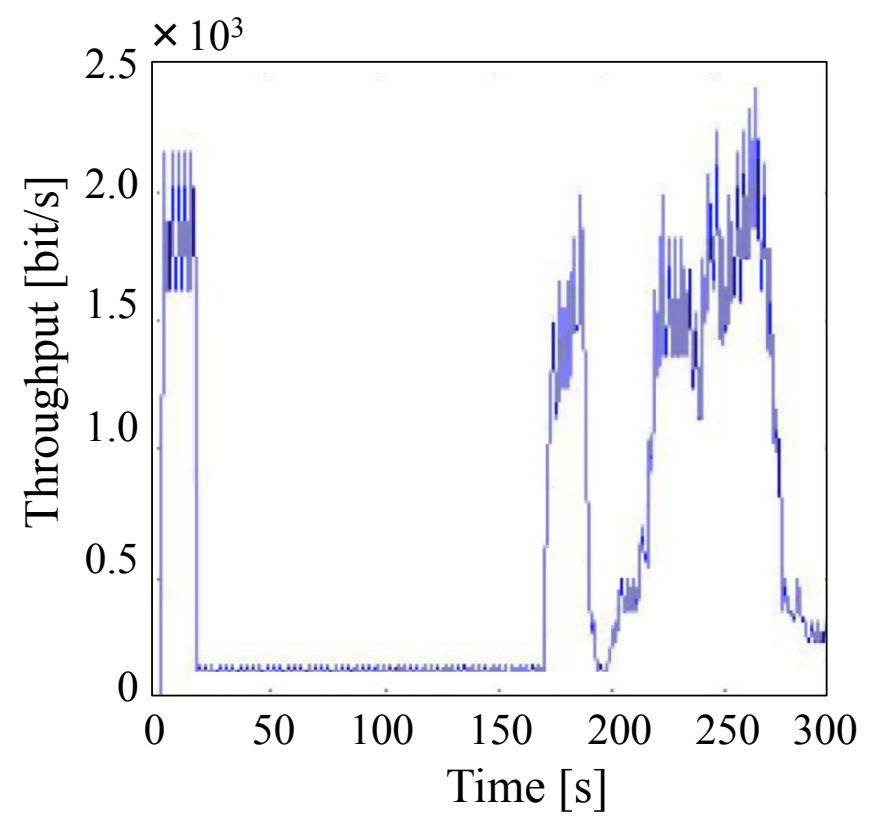

(b)

Figure 10: Highway scenario. Average throughput for (a) SRB technique, and (b) broadcast approach, vs. simulation time. 